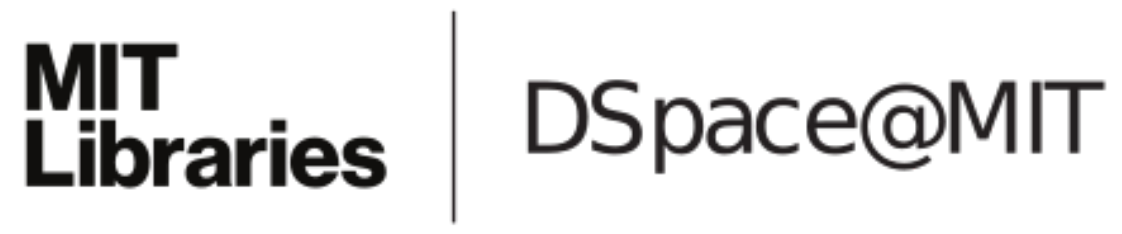

\author{
MIT Open Access Articles
}

Cross section measurements with monoenergetic muon neutrinos

The MIT Faculty has made this article openly available. Please share how this access benefits you. Your story matters.

Citation: Spitz, J. "Cross Section Measurements with Monoenergetic Muon Neutrinos." Phys. Rev. D 89, no. 7 (April 2014). ( 2014 American Physical Society

As Published: http://dx.doi.org/10.1103/PhysRevD.89.073007

Publisher: American Physical Society

Persistent URL: http://hdl.handle.net/1721.1/89015

Version: Final published version: final published article, as it appeared in a journal, conference proceedings, or other formally published context

Terms of Use: Article is made available in accordance with the publisher's policy and may be subject to US copyright law. Please refer to the publisher's site for terms of use. 


\title{
Cross section measurements with monoenergetic muon neutrinos
}

\author{
J. Spitz \\ Massachusetts Institute of Technology, Cambridge, Massachusetts 02139, USA
}

(Received 24 February 2014; published 8 April 2014)

\begin{abstract}
The monoenergetic $236 \mathrm{MeV}$ muon neutrino from charged kaon decay at rest $\left(K^{+} \rightarrow \mu^{+} \nu_{\mu}\right)$ can be used to produce a novel set of cross section measurements. Applicable for short- and long-baseline acceleratorbased neutrino oscillation experiments, among others, such measurements would provide a "standard candle" for the energy reconstruction and interaction kinematics relevant for charged current neutrino events near this energy. This neutrino can also be exercised as a unique known-energy, purely weak interacting probe of the nucleus. A number of experiments are set to come online in the next few years that will be able to collect and characterize thousands of these events.
\end{abstract}

DOI: 10.1103/PhysRevD.89.073007

PACS numbers: $14.60 . \mathrm{Pq}, 13.15 .+\mathrm{g}$

\section{INTRODUCTION}

The muon neutrino charged current $\left(\nu_{\mu} \mathrm{CC}\right)$ interaction is central to accelerator-based neutrino physics. However, despite the recent rapid progress in detection technology and analysis techniques, it is still quite difficult to measure the energy of $\nu_{\mu} \mathrm{CC}$ events, especially in the case of an interaction with a nuclear target. Final state interactions (FSIs), Fermi momentum, short-range correlations between nucleons, and the usually undetectable potential outgoing nuclear deexcitation gammas and neutrons can all work to convolute the reconstructed neutrino energy. This is before detector limitations, such as resolution, blindness to hadrons due to (e.g.) Cerenkov threshold, and event classification errors or ambiguity are even considered. Although a detector sensitive to the low-energy hadronic component of the final state can alleviate this at some level, many of these complications are simply inherent to the neutrino-nucleus system itself. As an example, a perfect detector's reconstruction of a $1 \mathrm{GeV} \nu_{\mu}$-nucleus $\mathrm{CC}$ quasielastic (CCQE, $\nu_{\mu} n \rightarrow \mu^{-} p$ ) event in terms of muon kinematics only provides an expected neutrino energy resolution of $\sim 20 \%$, with significant non-Gaussian asymmetric tails on either side of the true energy [1-4]. Although there are a number of predictions for the spectral smearing due to nuclear effects and the general direction of the convolution is understood, it is still highly nontrivial to correctly transform a reconstructed neutrino energy distribution into a true energy distribution with proper correlations and uncertainty estimates. Differences between neutrino and antineutrino events in terms of the nuclear physics that affects each can also be difficult to quantify.

A substantial amount of experimental effort has recently been directed toward measuring $\mathrm{CC}$ and neutral current (NC) neutrino cross sections at the hundreds of $\mathrm{MeV}$ and $\mathrm{GeV}$ scale $[5,6]$. These measurements are interesting for the study of the neutrino-nucleus interaction itself, the neutrino as a probe of the nucleus (e.g. the strange spin component of the nucleon, $\left.\Delta_{s}[7,8]\right)$, and, perhaps most importantly, are essential for long-baseline neutrino oscillation programs and informing the simulations [9-11] that such experiments rely on. The interaction cross section has also garnered a great deal of interest recently from theorists at the intersection of neutrino and nuclear physics, especially in the context of meson exchange currents and short-range correlations between nucleons [12-18].

The decay at rest of a positively charged kaon $\left(K^{+} \rightarrow \mu^{+} \nu_{\mu}, \mathrm{BR}=63.6 \%\right.$ [5]) produces a $236 \mathrm{MeV}$ muon neutrino. A large sample of these monoenergetic neutrinos, interacting via the $\mathrm{CC}$ channel $\nu_{\mu}{ }^{12} \mathrm{C} \rightarrow \mu^{-} X$, where $X$ is a proton and/or an excited nucleus, can be recorded to produce a standard candle for the $\nu_{\mu}$ and its interaction kinematics at and near this energy. As discussed in Ref. [19], these charged kaon decay-at-rest neutrinos can also be used for a sensitive probe of high- $\Delta m^{2}$ oscillations indicative of a sterile neutrino. Unfortunately, these measurements are only really possible with neutrinos, rather than both neutrinos and antineutrinos, because of nuclear capture for negatively charged kaons.

This article serves to point out the importance of measuring the differential and total cross sections associated with this unique, monoenergetic neutrino. For intense kaon decay-at-rest neutrino sources in general, a determination of the monoenergetic $\nu_{\mu}$ flux is complicated as the kaon production rate is difficult to accurately simulate and measure. Therefore, a precision, absolutely normalized cross section measurement is challenging. As discussed later, however, an exclusive interaction channel (for a carbon target, at least) can be used to determine the flux at the $10 \%$ level in the case that enough events are collected. Either way, a single differential or double differential cross section measurement in terms of outgoing muon angle and/or momentum, with detailed shape information, is valuable. Given a known neutrino energy, a precision differential cross section measurement in energy transfer, as is common for electron scattering experiments, is also available. No such measurement exists for $\nu_{\mu} \mathrm{CC}$ 
interactions. After discussing the relevance of these measurements in a number of physics applications, a set of potential experimental locations and detection technologies are considered.

\section{PHYSICS WITH NEUTRINOS FROM KAON DECAY AT REST}

Along with using the known-energy neutrinos as a unique probe of the nucleus, and as a test of our theoretical description of neutrino-nucleus interactions at this energy, a set of $236 \mathrm{MeV} \nu_{\mu} \mathrm{CC}$ cross section measurements can be utilized in a number of ways.

Long-baseline neutrino oscillation experiments employ near and far detectors for measuring the $L / E$-dependent mixing probability of a beam of originally $\nu_{\mu}$ or $\bar{\nu}_{\mu}$. These experiments are able to run in either neutrino or antineutrino mode by changing the polarization of their beamline magnets to focus $\pi^{+}\left(\rightarrow \mu^{+} \nu_{\mu}\right)$ or $\pi^{-}\left(\rightarrow \mu^{-} \bar{\nu}_{\mu}\right)$. The near detector, typically hundreds of meters from the source, provides a description of the initial, preoscillation composition of the mostly pure $\nu_{\mu}$ or $\bar{\nu}_{\mu}$ beam. The far detector, typically hundreds of kilometers from the source, probes the beam for $\nu_{\mu}$ and $\bar{\nu}_{\mu}$ disappearance as well as $\nu_{e}$ and/or $\nu_{\tau}$ appearance. These experiments are sensitive to the $\theta_{23}$ octant, the orientation of the mass hierarchy, and the neutrino $C P$-violating phase $\delta_{C P}$, among other physics.

The $\nu_{\mu}$ or $\bar{\nu}_{\mu} \mathrm{CCQE}$ interaction is often utilized both as the signal channel for the disappearance measurements and to constrain predictions relevant for the appearance channels. Given the $L / E$ oscillation dependence, reconstructing the energy of these neutrinos is obviously vital to extracting the mixing parameters. Further, an appropriate comparison between the near and far detector event rate as a function of reconstructed neutrino energy (and/or lepton kinematics, as in Ref. [20]) requires knowledge of the interaction cross section because the energy composition of the beam is different at each site, even in the case that both detectors are on axis. Along with near-far flux differences due to oscillations, this is due to the fact that the near detector is exposed to a range of incident neutrino angles (and corresponding kinematics) from pions decaying in flight at different locations in the decay pipe, while the far detector is effectively exposed to a point source of neutrinos. The possibility of systematic differences between the near and far sites in composition, size, detection technology, etc., can lead to further reliance on knowledge of the underlying cross section as well.

As an example, the uncertainties associated with the neutrino interaction dominate the systematics on the predicted number of signal $\nu_{e}$ events in the T2K longbaseline neutrino oscillation experiment $[20,21]$. These uncertainties, especially those related to the $\nu_{\mu} / \nu_{e}$ $\left(\bar{\nu}_{\mu} / \bar{\nu}_{e}\right)$ cross section ratio [22], will likely be among the leading sources of systematic uncertainties in future $\delta_{C P}$ programs, and there are multiple experiments around the world with the goal of improving our knowledge of the kinematics of the outgoing charged lepton and the neutrino/ antineutrino cross section on various nuclear targets in the long-baseline energy regime. The situation is such that it may be advantageous for future long-baseline experiments to tune their characteristic neutrino fluxes to better align with regions of low cross section and/or energy reconstruction uncertainty.

The few-hundreds-of-MeV neutrino energy range is an interesting possible place of emphasis for future longbaseline experiments for a number of reasons. Along with tuning closer to the second or third oscillation maxima, depending on baseline, in an attempt to better distinguish between different $\delta_{C P}$ scenarios and perhaps moving toward a more well-understood cross section and neutrino energy reconstruction region, given a precise set of (e.g.) $236 \mathrm{MeV}$-based measurements, emphasizing a lower beam energy can also prove advantageous because of the smaller resonant background and reduction of complications due to short-range correlations. Short-range correlations are not expected to play a large role at these relatively low neutrino energies [2,3]. However, in the case of a statistics-limited measurement, as compared to a background- or interactionsystematics-dominated one, a lower energy may not be optimal due to the reduction in cross section, and therefore event rate, as well as the lack of muon reconstruction abilities below $54 \mathrm{MeV}$ for water-based Cerenkov detectors such as SuperK/HyperK. Further, it is not clear if the benefit of the cross section measurements outlined here, in a move to lower energy, can outweigh the comparatively poor theoretical understanding of the $\nu_{\mu} / \nu_{e}$ cross section ratio in this low energy part of the $\mathrm{QE}$ regime.

Beyond the general comments above, the impact of a set of $236 \mathrm{MeV}$ cross section measurements is potentially wide ranging, especially in the case of a future experiment that relies on cross section knowledge in the few-hundred-MeV neutrino energy region. As an example, the European Spallation Source Neutrino Super Beam (ESS LSB) longbaseline neutrino oscillation project aims to produce a $5 \mathrm{MW}, 2 \mathrm{GeV}$ proton beam and combine it with a water Cerenkov based detector $300-600 \mathrm{~km}$ away in order to measure $\delta_{C P}$ [23]. In neutrino mode, the $\nu_{\mu}$ flux peaks at about $225 \mathrm{MeV}$. There is also an idea to employ simultaneous, high power 8 - and $60-\mathrm{GeV}$ proton beams with a $200 \mathrm{kt}$ water Cerenkov detector to obtain sensitivity to "low" energy $(0.2-1.5 \mathrm{GeV}) \nu_{\mu} \rightarrow \nu_{e}$ oscillations at the second oscillation maximum at a distance of $1300 \mathrm{~km}$. Such an experiment would provide a precise determination of $\delta_{C P}$ that is largely independent of the mass hierarchy orientation [24]. A cross section measurement with kaoninduced neutrinos is also quite relevant for a future $\beta$ beam with a $\gamma$ near 100 and/or a $3.5 \mathrm{GeV}$ Super Proton Linac (SPL) superbeam from CERN; both sources will produce neutrino fluxes in the few-hundred-MeV range [25]. 
The $\beta$-beam-SPL combination has been identified as a future option that will have greater sensitivity to $\delta_{C P}$ than any other superbeam or $\beta$-beam concept, second only to a neutrino factory [26].

As is true for all neutrinos in the long-baseline energy regime, nuclear effects play a large role for $236 \mathrm{MeV} \nu_{\mu}$ interactions and, despite the value in the cross section measurements outlined, it should be stated that this neutrino energy is a challenging one to deal with theoretically. The neutrino energy, or rather the characteristic energy transfer, is right at the transition between our neutrino-on-nucleus and neutrino-on-nucleon frameworks. The impulse approximation, in which it is assumed that the neutrino interacts with a single nucleon, breaks down at these lower energies and the distinction between a pre-FSI pure CCQE interaction $\left(\nu_{\mu} n \rightarrow \mu^{-} p\right)$ and an "absorption" interaction (on carbon, for example, $\nu_{\mu}{ }^{12} \mathrm{C} \rightarrow \mu^{-} X$ ) becomes blurred. Indeed, measurements of the monoenergetic neutrino may shed light on this important transition and inform the theoretical representations.

\section{EXPERIMENTAL LOCATIONS}

There are a number of proton fixed target experimental locations around the world where these cross section measurements are possible. The main requirement, other than a capable existing or planned detector, is that the primary proton energy exceed $\sim 3 \mathrm{GeV}$ for adequate kaon production. The 1.4 MW, $1 \mathrm{GeV}$ Spallation Neutron Source at Oak Ridge and the future $5 \mathrm{MW}, 2 \mathrm{GeV}$ European Spallation Source are therefore not considered here. In addition, given that a decay-at-rest source produces an isotropic flux of neutrinos, the detector needs to be reasonably close $(\lesssim 100 \mathrm{~m})$ to the source. The NuMI beam dump at Fermilab and the JPARC Materials and Life Science Facility (MLF) spallation source, in combination with a set of nearby planned detectors, are considered as possible experimental locations here.

The $120 \mathrm{GeV}$ NuMI beam line terminates for all nonneutrinos at a beam dump, $720 \mathrm{~m}$ downstream of the target [27]. Given the target's two interaction lengths, as much as $14 \%$ of the beam is passed on to this beam stop. The NuMI beam dump therefore provides a significant source of kaon decay-at-rest neutrinos and there are a set of nearby detectors that are sensitive to the $\nu_{\mu} \mathrm{CC}$ interaction at $236 \mathrm{MeV}$ : MiniBooNE, running since 2002 and located about $85 \mathrm{~m}$ from the NuMI dump, is a Cerenkov- and scintillation-based mineral oil detector [28], and MicroBooNE, running in 2014 and located about $102 \mathrm{~m}$ from the dump, is a liquid argon time projection chamber (LArTPC) [29]. While MiniBooNE has probably collected thousands of these from-NuMI events [30], it is likely difficult to unambiguously identify them as monoenergetic ones given the detector's propensity for lepton-only reconstruction for this class of interactions. Further, the pion decay-in-flight $\nu_{\mu}$ "background," largely coming from the NuMI decay pipe, likely makes the bump difficult to pick out. However, MiniBooNE+, an experimental proposal to add liquid scintillator to the existing MiniBooNE detector [31], may be able to enhance the energy reconstruction abilities enough to resolve the from-kaon peak. With full kinematic reconstruction abilities, as with the MicroBooNE LArTPC, the decay-in-flight background can be reduced significantly with reconstructed neutrino energy and direction requirements; this experiment is an attractive future location for these measurements.

It is worth noting that the SciBooNE experiment has also likely collected a significant number of these monoenergetic neutrinos, originating at the booster neutrino beam line (BNB) dump, but the decay-in-flight $\nu_{\mu}$ flux from the BNB decay pipe is more than an order of magnitude higher in the relevant energy region [32]. This will also be true for the future LAr1-ND experiment [33], proposed to be located at the SciBooNE hall in the BNB.

The JPARC-MLF is host to another intense source of kaon decay-at-rest neutrinos coming from $3 \mathrm{GeV}$ protons on a mercury target. The eventually $1 \mathrm{MW}$ source features only a small decay-in-flight background component as it is nominally used for spallation neutron production rather than as a conventional neutrino beam line. The mercury target is basically surrounded on all sides by concrete and iron, and the large majority of pions, muons, and kaons created quickly come to rest and capture or decay. Currently, there are plans to place a 50 ton fiducial volume gadolinium-loaded liquid scintillator (LS) neutrino detector $17 \mathrm{~m}$ from the source for a sterile neutrino search there [34]. Such a detector can also be used to perform the measurements described here.

\section{DETECTION}

We survey the detection technologies associated with the JPARC-MLF (LS) and MicroBooNE (LArTPC) experiments when considering these cross section measurements. Although a Cerenkov-based detector with muon-only reconstruction proficiency could potentially pick out the monoenergetic bump in reconstructed energy due to the $236 \mathrm{MeV}$ neutrino, especially in the absence of a significant nonmonoenergetic background, both technologies considered here have better neutrino energy reconstruction capabilities, mainly because of their ability to reconstruct the low-energy nucleonic component of these events; LS and LArTPC technology are simply more suitable for making sure that the events being evaluated are indeed coming from charged kaon decay at rest. Of course, what is learned from these measurements as well as their applicability toward future oscillation programs is highly dependent on which nuclear target is chosen.

The detection of $\nu_{\mu}$ CC events up to $\sim 260 \mathrm{MeV}$ with LS in the Liquid Scintillator Neutrino Detector (LSND) experiment is discussed at length in Ref. [35]. A combination of both 
scintillation and Cerenkov light signals can provide directional, calorimetric, and particle identification information for reconstructing the events. The muon is identified by requiring a delayed coincidence with a characteristic decay electron and can also be distinguished with a Cerenkov signal since nearly $90 \%$ of monoenergetic events produce a muon above the $36 \mathrm{MeV}$ kinetic energy threshold (in the commonly used linear alkyl-benzene based LS). Stopping $\mu^{-}$are captured $8 \%$ of the time on carbon in the LSND detector [35]. A veto, in combination with beam timing, can render the steady state background, mainly coming from cosmic ray muon decay in the detector, negligible. Notably, the JPARCMLF source features an extremely tight beam window with two $80 \mathrm{~ns}$ wide pulses of protons $540 \mathrm{~ns}$ apart at $25 \mathrm{~Hz}$, resulting in a steady state rejection factor of $4 \times 10^{-6}$. It is also expected that the JPARC-MLFLS detector will feature faster electronics than LSND, although it is difficult to estimate the achievable muon momentum and angular resolutions until the detector parameters, such as photocoverage and time resolution, are finalized. For reference, LSND's 25\% photocoverage resulted in a muon directional reconstruction resolution of about $12^{\circ}$ for muons above threshold and an energy resolution of better than $10 \%$ at $T_{\mu}=$ $100 \mathrm{MeV}[35,36]$.

In a best case scenario, the contributions of the scintillation- and (usually) Cerenkov-ring-producing muon and scintillation-only proton (or protons, since FSIs and correlations can result in multiple ejected nucleons), can be separated in LS for a more precise measurement of the differential cross sections, especially in terms of reconstructing the momentum of the muon. In practice, however, this is difficult and will likely require successfully modeling the light production of both the outgoing proton(s) and nuclear deexcitation gammas.

In a LArTPC, the charged particles created in a neutrino interaction, the reconstruction of which is required in order to infer the energy and flavor of the neutrino itself, propagate through the liquid argon medium and create trails of ionization along their paths. An electric field is imposed in the liquid argon volume and the trails are drifted through the noble liquid toward a set of sensing electrodes. The signals in time captured by the electrodes, usually in the form of a set of wire planes oriented at an angle with respect to one another, provide a complete threedimensional image of the neutrino event. Calorimetric information is available as the ionization collected by the electrodes is related to the amount of energy deposited along the charged particle tracks. Scintillation light $(128 \mathrm{~nm})$ is also produced readily as the charged particles ionize atoms; argon's high scintillation yield is useful for detecting this aspect of the interaction as well, although a wavelength shifter is required in conjunction with photomultiplier tubes to shift the light into the visible spectrum and detect it. With sensitivity to deexcitation gammas, neutrons, protons down to the few-tens-of-MeV level, and precise calorimetric reconstruction abilities, LArTPC technology is attractive for detecting and characterizing $236 \mathrm{MeV} \nu_{\mu} \mathrm{CC}$ events.

Table I shows the expected number of monoenergetic $\nu_{\mu}$ $\mathrm{CC}$ events in both MicroBooNE and the LS detector at the JPARC-MLF. The MicroBooNE event rate estimate assumes 2 years of running NuMI in neutrino mode at $700 \mathrm{~kW}\left(6 \times 10^{20} \mathrm{POT} /\right.$ year $)$, consistent with the Fermilab road map. Interestingly, NuMI neutrino mode and antineutrino mode each provide a similar flux of monoenergetic neutrinos. The JPARC-MLF event rate estimate assumes 4 years of running with a 1 MW beam and 4000 hours/year of operation, or $3 \times 10^{22}$ POT/year, consistent with Ref. [34]. The neutrino flux at each location has been determined using GEANT4 [37] (and FLUKA [38] also, in the case of NuMI) simulations of the sources, noting that kaon production is highly uncertain at both locations. As an example, the kaon-induced monoenergetic $\nu_{\mu}$ production at the $3 \mathrm{GeV}$ JPARC-MLF source is 0.0035 $\nu_{\mu} /$ proton with GEANT4 but is found to be about $75 \%$ higher with the LAQGSM/MARS (MARS15) software package [39]. The GEANT4 results are used here in order to be conservative. The event rate estimates also assume a $\nu_{\mu}$ CC cross section of $1.3 \times 10^{-39} \mathrm{~cm}^{2} /$ neutron, consistent with the NuWro neutrino event generator for interactions on both carbon and argon at $236 \mathrm{MeV}$ [9] and the theoretical predictions [40]. The expected neutrino flux from the JPARC-MLF source in all directions, without regarding potential detector location, in the energy range $100-300 \mathrm{MeV}$ can be seen in Fig. 1. The $236 \mathrm{MeV} \nu_{\mu}$ and three-body kaon decay " $K_{e 3}^{+}$" $\left(K^{+} \rightarrow \pi^{0} e^{+} \nu_{e}, \mathrm{BR}=5.1 \%\right)$ $\nu_{e}$ distributions are obviously quite prominent.

The NuWro neutrino event generator has been used here in order to simulate $236 \mathrm{MeV} \nu_{\mu} \mathrm{CC}$ interactions on carbon and argon. The simulation provides an idea of what can be expected from these neutrinos, although the employed impulse approximation is known to simulate neutrinos, especially $\nu_{\mu}$, poorly at these relatively low energies. NuWro is used because it contains a spectral-function-based

TABLE I. The expected monoenergetic $\nu_{\mu} \mathrm{CC}$ event rate at two experimental locations along with the beam exposure and detector assumptions.

\begin{tabular}{lccrr}
\hline \hline Detector (source) & Target (mass) & Exposure & Distance from source & $236 \mathrm{MeV} \nu_{\mu} \mathrm{CC}$ events \\
\hline MicroBooNE (NuMI dump) & LAr (90 ton) & $1.2 \times 10^{21}$ POT (2 years) & $102 \mathrm{~m}$ & 2300 \\
Liq. scint. (JPARC-MLF) & Gd-LS (50 ton) & $1.2 \times 10^{23}$ POT (4 years) & $17 \mathrm{~m}$ & 194000 \\
\hline \hline
\end{tabular}




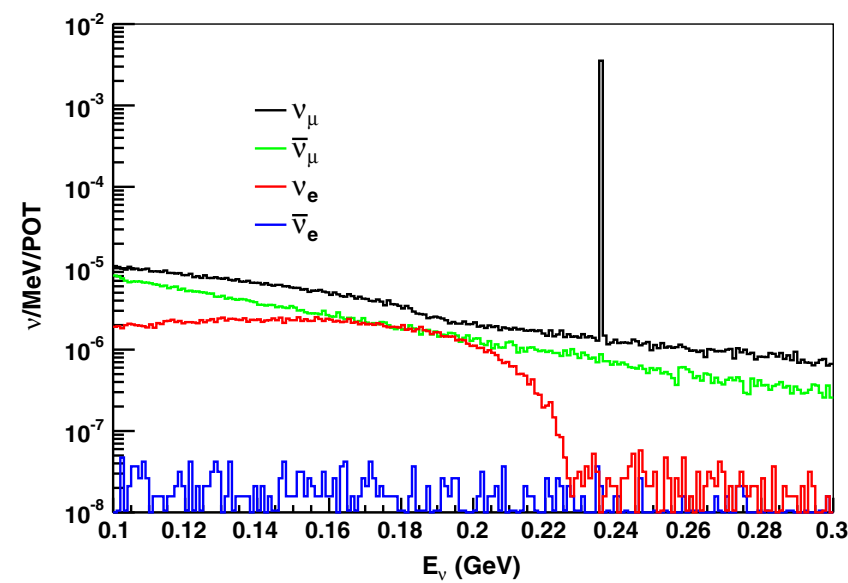

FIG. 1 (color online). The neutrino flux from 100-300 MeV provided by the $3 \mathrm{GeV}$ proton-on-mercury JPARC-MLF source. The $236 \mathrm{MeV}$ charged kaon decay-at-rest daughter $\nu_{\mu}$ is easily seen.

simulation for both nuclei [41]. The results of the neutrinoon-carbon simulation are shown in Fig. 2. The kinetic energy of the muon is seen along with a Gaussian smeared energy, given an arbitrary $10 \%$ detection resolution. Also, the post-FSI reconstructed neutrino energy $\bar{E}_{\nu}$ $\left(=E_{\mu}+\sum_{i}^{n} T_{i \text {,proton }}+S_{p}\right.$, where $n$ is the number of protons and $S_{p}=16 \mathrm{MeV}$ is the proton separation energy for ${ }^{12} \mathrm{C}$ ) with a perfect detector, after considering neutron and deexcitation gammas nonreconstructable, is shown. The separation energy for a single proton only is used for simplicity. The apparent bimodal shape of the distribution is due to the shell structure of the nucleus and the energy levels of the neutron within the spectral function

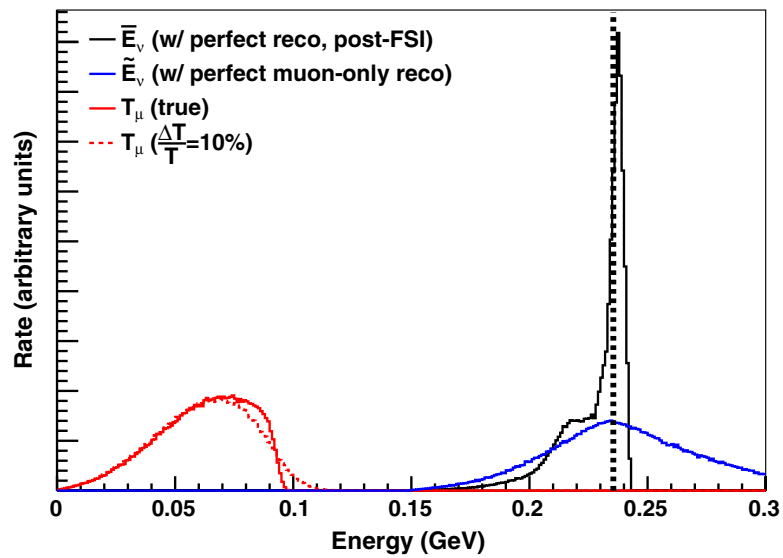

FIG. 2 (color online). The NuWro simulation results of $236 \mathrm{MeV} \nu_{\mu}$ (from $K^{+} \rightarrow \mu^{+} \nu_{\mu}$ ) CC interactions on carbon. The kinetic energy of the outgoing muon, given a set of detector resolution assumptions, is shown. The reconstructed neutrino energy available with a perfect detector is also visible, noting that deexcitation gammas and neutrons are considered missing energy here. The reconstructed neutrino energy with perfect muon-only tracking is also shown for reference. implementation. The reconstructed energy with a perfect muon-only detector $\tilde{E}_{\nu}$, given the usual two-body kinematics CCQE formula, assuming target nucleon at rest and a binding energy of $34 \mathrm{MeV}$, is also shown for reference. The shape of the expected $236 \mathrm{MeV} \nu_{\mu} \mathrm{CC}$ event rate distribution in muon angle and kinetic energy, as simulated with NuWro, is shown in Fig. 3.

The main requirement for a valuable monoenergetic $\nu_{\mu}$ cross section measurement is that the signal interaction is properly identified as such. This determination relies on the ability to precisely reconstruct the energy of the neutrino, with the actual energy resolution needed depending on the background nonmonoenergetic $\nu_{\mu}$ flux in the energy region of interest. In the case of the JPARC-MLF source, for example, the monoenergetic cross-section-weighted flux, without regard for detector location, will be a factor of $\sim 30$ times higher than the integrated background in an arbitrary true energy window of $80 \mathrm{MeV}$ around $236 \mathrm{MeV}$. The actual signal to background at the detector location will likely be significantly higher than this, given the tentative backward orientation of the detector relative to the primary proton beam direction, according to Ref. [34], and resulting decrease in the decay-in-flight component at the detector. For example, the ratio increases to $\sim 180$ in the case that only neutrinos with $\cos \theta_{z}<0$ (where $+z$ is the primary proton direction) are considered. Regardless, if a $\nu_{\mu} \mathrm{CC}$ event is identified from this source, with even modest energy resolution, one can be fairly confident that it is monoenergetic.

The favorable signal-to-background ratio at the JPARCMLF may afford the ability to perform these cross section measurements with a water Cerenkov detector, capable of providing lepton-only kinematic reconstruction, for a cross section measurement directly applicable to the Super-K and Hyper-K detectors [42] within the T2K long-baseline program, and water-based devices in general. However, the $236 \mathrm{MeV} \nu_{\mu}$ is at the low end of neutrino energies relevant for $\mathrm{T} 2 \mathrm{~K}\left(E_{\nu}^{\mathrm{peak}} \sim 600 \mathrm{MeV}\right)$. Further, the water

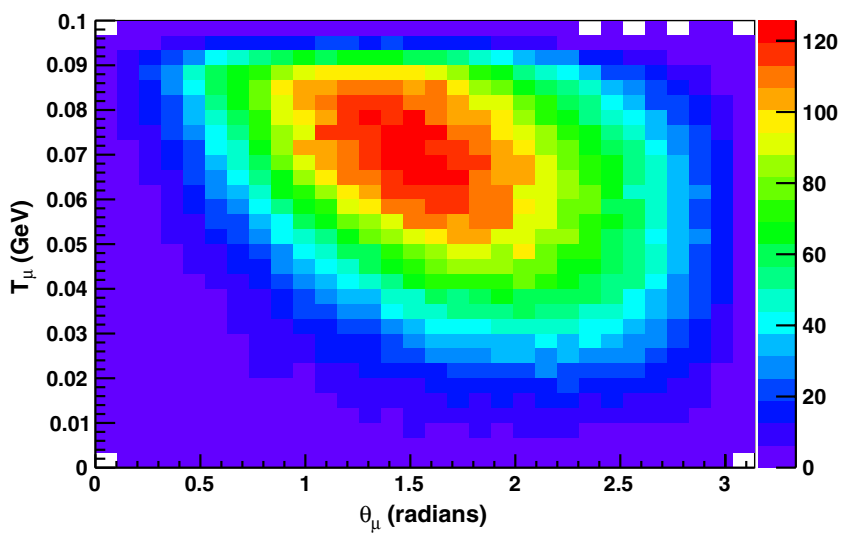

FIG. 3 (color online). The kinematics of the muon from $236 \mathrm{MeV} \nu_{\mu} \mathrm{CC}$ interactions on carbon according to NuWro. The $z$-axis units are arbitrary. 
Cerenkov threshold for muons is $54 \mathrm{MeV}$ in kinetic energy so one-third of the signal is nominally irretrievable (see Fig. 2) and, when considering muons in the T2K $\nu_{\mu}$ disappearance analysis, Super-K's threshold (200 MeV/c, due to particle identification requirements at low energy) is above the characteristic momenta for monoenergetic events [43].

The large JPARC-MLF signal-to-background ratio in an arbitrary true neutrino energy window of $80 \mathrm{MeV}$ around $236 \mathrm{MeV}$ decreases to $\sim 1$ in the case of the NuMI beam dump source given the substantial pion decay-in-flight background $\nu_{\mu}$ flux. Along with precisely reconstructing the neutrino energy in order to reduce background, this issue can be mitigated somewhat with the ability to reconstruct the direction of the incoming neutrino since the large majority of monoenergetic $\nu_{\mu}$ will be coming directly from the dump rather than the NuMI decay pipe or target station. This requires the ability to reconstruct the low-energy hadronic component of the interaction at a reasonable level as the outgoing muon and incoming neutrino direction are rather poorly correlated. Notably, the ArgoNeuT LArTPC detector has demonstrated the ability to reconstruct protons down to $21 \mathrm{MeV}$ kinetic energy [44]. While it is currently not clear exactly how well MicroBooNE will be able to reconstruct the direction of $236 \mathrm{MeV}$ events, especially given that nuclear effects can distort the direction of the outgoing nucleon(s), the detector's orientation relative to the NuMI beam line and dump, with the NuMI target and dump separated by about $110^{\circ}$, will make it easier to distinguish between neutrinos from the different sources. Even if this is not achievable, the decay-in-flight background contribution to a monoenergetic $\nu_{\mu}$ measurement can be constrained by considering events outside of the relevant energy region. Data collection with a proton beam plug or lengthened target, which would substantially reduce the monoenergetic flux at MicroBooNE, could also allow for an in situ measurement of the background component.

\section{OTHER OPPORTUNITIES WITH KAON DECAY-AT-REST NEUTRINOS}

Aside from measurements of the $\nu_{\mu} \mathrm{CC}$ differential and total cross sections described above, from-kaon neutrinos provide a number of other physics opportunities as well. Two of these are described below: (i) A measurement of an exclusive channel can deliver a precise determination of the neutrino flux at $236 \mathrm{MeV}$, and (ii) the charged kaon decay $K_{e 3}^{+}$can provide a significant sample of $\nu_{e}$ events in an energy range relevant for accelerator-based oscillation measurements.

Despite the complications discussed above with regard to the transition between neutrino-on-nucleon and neutrinoon-nucleus scattering, the contribution from the absorption part of the inclusive interaction can be seen as something of a windfall, at least when considering a carbon target, given the significant $\nu_{\mu}{ }^{12} \mathrm{C} \rightarrow \mu^{-12} \mathrm{~N}_{\mathrm{gs}}$ exclusive cross section at this energy. This exclusive reaction, which can be purely identified with a triple coincidence of the muon, the decay daughter electron, and the positron from the $\beta$ decay of ${ }^{12} \mathrm{~N}_{\mathrm{gs}}$ has a well-predicted cross section at $236 \mathrm{MeV}$ of $\approx 7 \times 10^{-41} \mathrm{~cm}^{2} /$ nucleus [45] which results in an event rate of about $1 \%$ relative to the inclusive channel. Although this rate is comparatively low, it still would provide nearly 2000 monoenergetic events in 4 years of running with the JPARC-MLF detector. While the theoretical cross section prediction for the inclusive channel is highly uncertain, with models differing by up to $25 \%$ for LSND's fluxaveraged cross section $\left(\left\langle E_{\nu}\right\rangle=156 \mathrm{MeV}\right)[35,40,46,47]$, the exclusive channel is well known, with differences between the various shell-model-based predictions at the level of only $10 \%$ at $250 \mathrm{MeV}[35,45]$. The exclusive prediction is more precise because it relies on form factors arrived at with measured values of the related electroweak transition probabilities ( $\beta$ decay and muon capture) [40]. Given the reliable cross section prediction, this exclusive channel can be used for the absolute flux determination at this energy and therefore for reporting precisely normalized differential and total cross section measurements.

Along with the monoenergetic $\nu_{\mu}$, kaons yield another potentially important source of neutrinos as well. While $\nu_{\mu}$ $\mathrm{CC}$ cross section measurements in the energy range 53-500 $\mathrm{MeV}$ are quite sparse [32,35,48,49], $\nu_{e}$ cross section measurements above $53 \mathrm{MeV}$ are nonexistent. A significant number of $\nu_{e} \mathrm{CC}$ events can be collected from the JPARC-MLF source (via $K_{e 3}^{+}$), noting that the $\nu_{e}$ cross section is about $25 \%$ higher than $\nu_{\mu}$ at these energies and the $\nu_{e}$ cross section has a significantly lower energy threshold due to the muon-electron mass difference. Although the $\nu_{e}$ are not monoenergetic, the flux does cut off sharply at $\approx 225 \mathrm{MeV}$ and has a characteristic energy shape. With regard to applying such a measurement to $\mathrm{T} 2 \mathrm{~K}$, the neutrino energy is low but well within relevance, given that $\mathrm{T} 2 \mathrm{~K}$ requires the reconstructed electron momentum to exceed $100 \mathrm{MeV} / c$ in their appearance analysis [20]. Indeed, one of T2K's $\nu_{e}$ appearance candidates has a reconstructed energy of $\approx 150 \mathrm{MeV}$ [20]. Since there are no $\nu_{e} \mathrm{CC}$ measurements at these energies and this channel, along with its antineutrino analog, represents the actual signal for a long-baseline neutrino oscillations experiment's $\delta_{C P}$ measurement, as well as numerous short-baseline electron-flavor appearance searches, this sample might prove quite useful.

About $6500 \nu_{e}$ CC events are expected from 100$225 \mathrm{MeV}$ in true neutrino energy in 4 years of running the JPARC-MLF 50-ton LS experiment. This estimate uses the $\nu_{e}$ cross section prediction from Ref. [46]. This sample may also be important as an experimental check of the $\nu_{\mu} / \nu_{e}$ cross section ratio, recalling that $\nu_{\mu}$ events are used to constrain the $\nu_{e}$ appearance expectation in both short- and long-baseline experiments. Notably, this ratio has been 


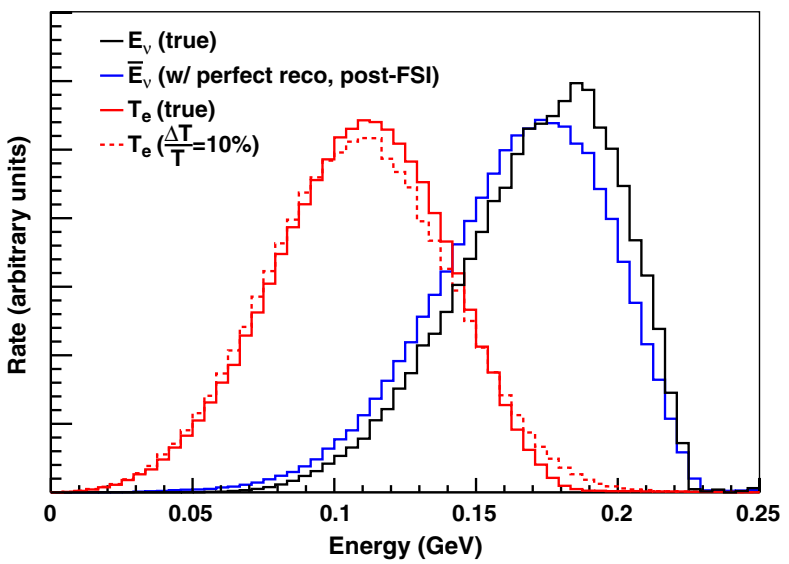

FIG. 4 (color online). The NuWro simulation results of $\nu_{e}$ (from $K^{+} \rightarrow \pi^{0} e^{+} \nu_{e}$ ) CC interactions on carbon. The kinetic energy of the outgoing electron, given a set of detector resolution assumptions, is shown. The true neutrino energy and reconstructed neutrino energy available with a perfect detector $\left(\bar{E}_{\nu}=E_{e}+\sum_{i}^{n} T_{i, \text { proton }}+S_{p}\right)$ are also visible.

identified as one of the keys to improving sensitivity to $\delta_{C P}$ in future long-baseline experiments [22] and our knowledge of it is weakest at low energy where the lepton mass difference and nuclear form factors contribute more. Measurements of these cross sections are also applicable in understanding the MiniBooNE low-energy excess at 250-475 MeV [50] and for informing MicroBooNE's study of this energy region and below, given the low(er) energy reconstruction capabilities of LArTPC technology. The results of a simulation of the $\nu_{e}$ from the JPARC-MLF source (shown in Fig. 1), which is basically the flux exclusively coming from $K^{+} \rightarrow \pi^{0} e^{+} \nu_{e}$, on a carbon target are shown in Fig. 4. The true neutrino energy, reconstructed neutrino energy available with a perfect detector $\bar{E}_{\nu}$ $\left(=E_{e}+\sum_{i}^{n} T_{i, \text { proton }}+S_{p}\right)$, and electron kinetic energy with two different detection resolution scenarios are shown. LS, LArTPC, and water Cerenkov technology are all capable of efficiently reconstructing $\nu_{e} \mathrm{CC}$ events in this energy range.
It is also worth briefly mentioning that monoenergetic $\nu_{\mu}$-induced NC events may offer an interesting physics sample as well, especially since NC measurements are always flux integrated; these may be useful for measuring $\Delta_{s}$ and/or isovector couplings in elastic scattering.

\section{CONCLUSION}

Measurements of the monoenergetic $236 \mathrm{MeV} \nu_{\mu}$ from charged kaon decay at rest may be quite valuable for probing the nucleus using neutrinos and accelerator-based oscillation experiments. A number of experiments coming online in the next few years, including MicroBooNE at Fermilab and the LS-based experiment at JPARC's MLF facility, will be able to make precise cross section measurements of this unique known-energy channel. In particular, the MLF experiment will see close to 200000 $236 \mathrm{MeV} \nu_{\mu} \mathrm{CC}$ events in 4 years of running along with $6500 \nu_{e}$ CC events coming from a well-understood flux shape in the 100-225 MeV range. In the future, it seems pertinent to develop a quantitative understanding of the role these measurements can play in reducing the systematics associated with both energy reconstruction and cross sections in accelerator-based oscillation experiments, and perhaps even informing decisions related to the development of these programs.

\section{ACKNOWLEDGMENTS}

The author wishes to thank J. Sobczyk and T. Golan for help with NuWro; J. M. Conrad for support and guidance; Z. Pavlovic and NOvA for the NuMI flux simulation used in the MicroBooNE event rate estimate; T. Maruyama and K. Nishikawa for providing a simplified geometry of the JPARC-MLF neutrino source that was used in the event rate estimate; T. Katori for valuable discussions and comments; and G. P. Zeller, F. Cavanna, M. J. Wilking, W. C. Louis, B. J. P. Jones, M. Moulai, and B. T. Fleming for comments on early drafts. The author is supported by a Pappalardo Fellowship in Physics at MIT and by the National Science Foundation under Grant No. PHY-1205175.
[1] M. Martini, M. Ericson, and G. Chanfray, Phys. Rev. D 85, 093012 (2012).

[2] M. Martini, M. Ericson, and G. Chanfray, Phys. Rev. D 87, 013009 (2013).

[3] J. Nieves, F. Sánchez, I. Ruiz Simo, and M. J. Vicente Vacas, Phys. Rev. D 85, 113008 (2012).

[4] O. Lalakulich, U. Mosel, and K. Gallmeister, Phys. Rev. C 86, 054606 (2012).

[5] J. Beringer et al. (Particle Data Group), Phys. Rev. D 86, 010001 (2012).
[6] J. A. Formaggio and G. P. Zeller, Rev. Mod. Phys. 84, 1307 (2012).

[7] A. A. Aguilar-Arevalo et al. (MiniBooNE Collaboration), Phys. Rev. D 82, 092005 (2010).

[8] A. A. Aguilar-Arevalo et al. (MiniBooNE Collaboration), arXiv:1309.7257.

[9] C. Juszczak, Acta Phys. Pol. B 40, 2507 (2009); T. Golan, C. Juszczak, and J. Sobczyk, Phys. Rev. C 86, 015505 (2012). NuWro version " $11 \mathrm{~m}$ " is used with a spectral function implementation. 
[10] C. Andreopoulos et al., Nucl. Instrum. Methods Phys. Res., Sect. A 614, 87 (2010).

[11] Y. Hayato, Acta Phys. Pol. B 40, 2477 (2009).

[12] J. E. Amaro, M. B. Barbaro, J. A. Caballero, T. W. Donnelly, and J. M. Udías, Phys. Rev. D 84, 033004 (2011).

[13] M. B. Barbaro et al., arXiv:1110.4739.

[14] A. Bodek and H. Budd, Eur. Phys. J. C 71, 1726 (2011).

[15] C. Giusti and A. Meucci, arXiv:1110.4005.

[16] M. Martini, M. Ericson, and G. Chanfray, Phys. Rev. C 84, 055502 (2011).

[17] J. Nieves, F. Sánchez, I. Ruiz Simo, and M. J. Vicente Vacas, J. Phys. Conf. Ser. 408, 012040 (2013).

[18] J. T. Sobczyk, Eur. Phys. J. C 72, 1850 (2012).

[19] J. Spitz, Phys. Rev. D 85, 093020 (2012).

[20] K. Abe et al. (T2K Collaboration), Phys. Rev. Lett. 112, 061802 (2014).

[21] K. Abe et al. (T2K Collaboration), Nucl. Instrum. Methods Phys. Res., Sect. A 659, 106 (2011).

[22] P. Huber, M. Mezzetto, and T. Schwetz, J. High Energy Phys. 03 (2008) 021.

[23] E. Baussan et al., arXiv:1309.7022.

[24] M. Bishai et al., arXiv:1307.0807.

[25] J. E. Campagne, M. Maltoni, M. Mezzetto, and T. Schwetz, J. High Energy Phys. 04 (2007) 003.

[26] P. Coloma, P. Huber, J. Kopp, and W. Winter, Phys. Rev. D 87, 033004 (2013).

[27] K. Anderson et al., Report No. FERMILAB-DESIGN1998-01, 1998.

[28] A. A. Aguilar-Arevalo et al. (MiniBooNE Collaboration), Nucl. Instrum. Methods Phys. Res., Sect. A 599, 28 (2009).

[29] H. Chen et al. (MicroBooNE Collaboration), Report No. FERMILAB-PROPOSAL-0974, 2007.

[30] P. Adamson et al., Phys. Rev. Lett. 102, 211801 (2009).

[31] R. Dharmapalan et al. (MiniBooNE+ ollaboration), arXiv:1310.0076.
[32] Y. Nakajima et al. (SciBooNE Collaboration), Phys. Rev. D 83, 012005 (2011).

[33] C. Adams et al., arXiv:1309.7987.

[34] M. Harada et al., arXiv:1310.1437.

[35] L. B. Auerbach et al., Phys. Rev. C 66, 015501 (2002).

[36] W. C. Louis (private communication).

[37] S. Agostinelli et al., Nucl. Instrum. Methods Phys. Res., Sect. A 506, 250 (2003).

[38] A. Ferrari et al., Reports No. CERN 2005-10, No. INFN/ TC_05/11, and No. SLAC-R-773, 2005.

[39] N. V. Mokhov, Report No. FERMILAB-FN-628, 1995; O. E. Krivosheev and N. V. Mokhov, Report No. FermilabConf-00/181, 2000; Report No. Fermilab-Conf-03/053, 2003; N. V. Mokhov et al., Report No. FermilabConf-04/053, 2004.

[40] C. Volpe, N. Auerbach, G. Colò, T. Suzuki, and N. Van Giai, Phys. Rev. C 62, 015501 (2000).

[41] O. Benhar, N. Farina, H. Nakamura, M. Sakuda, and R. Seki, Phys. Rev. D 72, 053005 (2005).

[42] K. Abe et al., arXiv:1109.3262.

[43] K. Abe et al. (T2K Collaboration), Phys. Rev. Lett. 111, 211803 (2013).

[44] T. Yang, for the ArgoNeuT Collaboration, arXiv:1311.2096.

[45] J. Engel, E. Kolbe, K. Langanke, and P. Vogel, Phys. Rev. C 54, 2740 (1996).

[46] E. Kolbe, K. Langanke, and P. Vogel, Nucl. Phys. A652, 91 (1999).

[47] A. C. Hayes and I. S. Towner, Phys. Rev. C 61, 044603 (2000).

[48] S. J. Barish et al., Phys. Rev. D 16, 3103 (1977).

[49] A. A. Aguilar-Arevalo et al. (MiniBooNE Collaboration), Phys. Rev. D 81, 092005 (2010).

[50] A. A. Aguilar-Arevalo et al. (MiniBooNE Collaboration), Phys. Rev. Lett. 102, 101802 (2009). 